\title{
Cardiac Evaluation prior to Kidney Transplantation
}

\author{
Rowena B. Delos Santos ${ }^{\star}$, Aleksandra Gmurczyk ${ }^{\dagger}$, Jagdeep S. Obhrai ${ }^{\ddagger}$, and Suzanne G. \\ Watnick \\ "Division of Nephrology and Hypertension, Department of Internal Medicine, Oregon Health and \\ Science University, Portland, Oregon \\ tDivision of Nephrology-Hypertension, Department of Internal Medicine, Northwestern University, \\ Chicago, Illinois
}

¥Transplant Medicine Section, Division of Nephrology and Hypertension, Department of Internal Medicine, Oregon Health and Science University, Portland, Oregon

\begin{abstract}
Kidney transplantation is the treatment of choice for most patients with stage 5 chronic kidney disease and end-stage renal disease (ESRD), offering improved quality of life and overall survival rates. However the limited supply of available organs makes this a scarce resource. Cardiovascular complications continue to be the leading cause of mortality in the kidney transplant population, accounting for over $30 \%$ of deaths with a functioning allograft. Thus, pre-operative cardiac risk assessment is critical to optimize patient selection and outcomes. Currently there is no consensus for cardiovascular evaluation in the chronic kidney disease and ESRD population prior to kidney transplantation; the recommendations of the American Society of Nephrology and American Society of Transplantation differ from those of the American Heart Association and the American College of Cardiology. Previously developed risk scores have also been used to risk stratify this population. In this review we discuss two cases that illustrate the difficulties of interpreting the prognostic value of current testing strategies. We also discuss the importance of different tests for cardiovascular evaluation as well as previous non-kidney transplant specific risk scores used in the pre-kidney transplant population.
\end{abstract}

Kidney transplantation is the treatment of choice for most patients with stage 5 chronic kidney disease (CKD) and end stage renal disease (ESRD), offering improved quality of life and overall survival rates (1). However, according to the Scientific Registry of Transplant Recipients (SRTR) at the end of 2008, there were 80,972 patients awaiting a kidney transplant. Of those, $20 \%$ were transplanted that year, while another $5.7 \%$ died while waiting for a transplant (2). Given this limited resource, it is important to try to limit transplantation to patients most likely to survive the procedure and to make optimal use of their allograft. Since cardiovascular complications account for over 30\% of deaths with a functioning allograft pre-operative cardiac risk assessment is critical to optimize patient selection and outcomes (3).

At present there is no consensus regarding the appropriate cardiac risk assessment strategy for CKD and ESRD patients awaiting kidney transplantation. The American Society of Nephrology (ASN) and the American Society of Transplantation (AST) recommend myocardial perfusion imaging as part of the pre-operative evaluation. Depending on test results, the patient could require revascularization (4). In comparison, the American College

Address correspondence to: Suzanne Watnick, MD, P3 NEPH, Associate Professor of Medicine, 3710 SW US Veterans Hospital Road, Portland VA Medical Center, Oregon Health \& Science University, Portland, OR 97239, Phone: 503-494-8490, Fax: 503-721-7810,watnicks@ohsu.edu. 
of Cardiology (ACC) and the American Heart Association (AHA) recommend no preoperative cardiac evaluation given that kidney transplantation poses an intermediate risk if the patient has good functional status, which we outline more fully in our later discussion (6). The lack of consensus leads to difficulty in determining which patients require no testing, non-invasive cardiac testing or invasive interventions prior to kidney transplantation. Here we discuss two relevant cases that illustrate the difficulty in predicting which candidates are at high risk for a cardiac event post-transplantation. We will also discuss the current methods of cardiac screening and various cardiac risk assessment strategies available.

\section{Patient 1}

Mr. M was a 62 year-old gentleman with stage 5 chronic kidney disease (CKD) due to autosomal dominant polycystic kidney disease (PKD) with an estimated glomerular filtration rate (eGFR) of $13 \mathrm{ml} / \mathrm{min} / 1.73 \mathrm{~m}^{2}$, not yet requiring dialysis. Table 1 describes the patient's baseline characteristics. On review of systems he reported some shortness of breath while trying to walk after a very large meal, different from his previous experience of angina that led to his cardiac stent placement. On exam, blood pressure was 118/72, pulse 67 beats per minute, and body mass index (BMI) of $29.6 \mathrm{~kg} / \mathrm{m}^{2}$. Significant findings on exam include a 2/6 systolic ejection murmur, mildly diminished pulses in the feet, no jugular venous distension, and mild pedal edema. His labs were remarkable with a hemoglobin of $10.8 \mathrm{~g} /$ $\mathrm{dL}$, cholesterol $148 \mathrm{mg} / \mathrm{dL}$, albumin $4.1 \mathrm{~g} / \mathrm{dL}$. His chest radiograph, EKG, cardiac stress test and echocardiogram showed no abnormalities. He did not have a cardiac catheterization. Mr. $\mathrm{M}$ underwent a living unrelated kidney transplant, and had immediate graft function.

The day after surgery he developed chest burning associated with EKG changes showing ST depression in V3-V6, and an elevation of cardiac troponin T levels to $0.27 \mathrm{ng} / \mathrm{dL}$, consistent with a non-ST elevation myocardial infarction. On post-operative day (POD) \#6, a cardiac catheterization showed 3-vessel coronary artery disease not amenable to cardiac stenting, and he subsequently underwent a coronary artery bypass surgery on POD \#19, and remains chest pain free 2 years later with stable renal function.

\section{Patient 2}

Ms. D was a 70 year old woman with ESRD due to diabetes. She had been on peritoneal dialysis for 6 months prior to kidney transplant evaluation. Table 1 describes the patient's baseline characteristics. On review of systems she had no shortness of breath, chest pain, palpitations or heart failure symptoms. She is able to climb stairs in her home without exhaustion.

On examination she had a blood pressure of 138/66, heart rate of 77 beats per minute, and body mass index of $22 \mathrm{~kg} / \mathrm{m}^{2}$. Significant findings on exam included a normal pulmonary and cardiovascular exam and trace lower extremity edema. Labs included a hemoglobin of $11.2 \mathrm{~g} / \mathrm{dL}$, cholesterol $168 \mathrm{mg} / \mathrm{dL}$ and albumin $2.4 \mathrm{~g} / \mathrm{dL}$. Her EKG shows normal sinus rhythm with nonspecific changes. A dipyridamole scan showed a small mild reversible apicolateral defect with an EF of 54\%. Due to the findings on the stress test, a cardiac catheterization was performed showing normal coronary arteries, left ventricular hypertrophy and ejection fraction of $77 \%$. She underwent an uncomplicated deceased donor kidney transplant 2 years later. She had one admission in the first month post-transplantation due to dehydration and diarrhea, but otherwise has been cardiovascular event free for four years post kidney transplantation. 


\section{Discussion}

These two cases illustrate one concern regarding kidney transplant outcomes, which is the ability to predict which recipients will have a cardiac event post-transplantation that would have been prevented with pre-transplant intervention. The goal of transplantation is not only the survival of the kidney allograft, but also the survival of the transplant recipient. Patient survival is most often threatened by cardiovascular disease, the most common cause of death in the transplant population (3). In a cohort of 7040 patients who died with graft function over a 10 year follow-up period, it was found that $36 \%$ died as a result of cardiovascular disease. In addition, $47 \%$ of those who died within 30 days of transplantation died of cardiovascular disease, particularly acute myocardial infarction (7). The ability to predict which candidates will have a cardiac event post-transplantation is a growing concern for transplant centers and their kidney transplant recipients. As illustrated in these two cases, one patient had significant cardiac disease that required intervention, which was not detected by routine screening methods. The other was thought to be at high risk for coronary disease but after undergoing catheterization, was not found to have disease. One could hypothesize that appropriate testing strategies and interventions prior to transplant surgery might have significantly changed outcomes.

\section{Current guidelines for screening}

There is consensus that cardiovascular assessment is needed in high-risk patients prior to kidney transplantation. However, optimal screening protocols and frequency of testing have not been fully established. The American College of Cardiology/ American Heart Association (ACC/AHA) recommend no cardiac evaluation prior to an intermediate risk surgery such as a kidney transplant if the patient's functional status is greater or equal to 4 metabolic equivalents without cardiac symptoms. A metabolic equivalent is the quantity of oxygen consumed while resting or sitting and is approximately $3.5 \mathrm{ml}$ of oxygen per kilogram of body weight per minute (5). Examples of activities equivalent to 4 metabolic equivalents include walking up a flight of stairs, walking at 4 miles per hour on level ground, heavy housework like scrubbing or moving furniture, and activities like bowling or golfing. If the person's functional status is not 4 or more metabolic equivalents, and if a person has a history of heart disease, heart failure, diabetes, cerebrovascular disease or renal disease, he/she can go forward with the operation with heart rate control, or consider noninvasive testing if the test will change management (6). These recommendations are based on a consensus of expert opinion after their thorough review of current scientific evidence.

These recommendations differ from the screening protocols supported by the American Society of Nephrology and American Society of Transplantation (ASN and AST). These two groups advocate non-invasive cardiac stress imaging, including stress echocardiography or nuclear myocardial perfusion testing for patients with diabetes, prior ischemic heart disease, or two of the following: men $>45$ years, women $>55$ years, ischemic disease in a first degree relative, smoking, hypertension, cholesterol $>200 \mathrm{mg} / \mathrm{dL}, \mathrm{HDL}<35 \mathrm{mg} / \mathrm{dL}$ or left ventricular hypertrophy. If the non-invasive testing is positive for ischemia, then the patient undergoes angiography for potential revascularization prior to transplantation (4).

Many transplant programs support the ASN guidelines. One rationale is the relatively high pre-test probability of cardiac disease in patients with chronic kidney disease compared to the general population (8-10). Up to half of asymptomatic ESRD patients initiating renal replacement therapy with no known cardiac disease have significant coronary artery disease on angiography (11). The progression of coronary disease also increases with increasing dialysis vintage In a cohort of ESRD patients who underwent at least 2 angiograms in a 12 60 month period, new coronary artery disease was diagnosed in 59\% of patients at the time 
of the first angiography, while $34 \%$ had no disease. Progression of coronary artery disease was found in $50 \%$ of patients at the second angiography. No patient had regression of coronary artery disease (12). Whether the more aggressive stance of the ASN/AST guidelines is superior to that of the ACC/AHA guidelines in reducing post-transplant events has never been prospectively examined.

\section{Cardiovascular Tests Pre-Transplant}

As the goal of pre-operative cardiac screening is to identify those who are at risk for having a peri-operative or post-operative event, it is reasonable to think that pre-operative intervention may potentially prevent future cardiac events. This section discusses the risks and benefits of different methods of cardiac testing pre-kidney transplant. Various methods have been used to evaluate cardiac disease in this population. Evaluation using SPECT thallium myocardial perfusion imaging was as good as planar imaging in predicting cardiac deaths and nonfatal cardiac events when trying to risk-stratify pre-transplant patients (13).

Over an average 28 month follow-up, the pre-transplant SPECT imaging was positive in all those who eventually had cardiac events, while those with normal SPECT imaging and risk factors had no cardiac events (13).

There have been mixed results regarding dipyridamole thallium perfusion studies with reported sensitivity ranging from $37 \%-80 \%$, while the specificity ranges from $37 \%-73 \%$ $(14,15)$. The wide range of sensitivity of a thallium test may be due in part to the nature of the testing, where the chemical agent thallium is detected differently on the scan depending on blood flow into the cardiac vessels and perfusion of the cardiac muscle. Hence, the presence of an equal amount of coronary plaque in the vessels may not result in perfusion defects detectable by this test.

Dobutamine stress echocardiographic testing has also been widely used in pre-transplant cardiac testing. An evaluation of several studies of ESRD patients undergoing dobutamine stress echocardiography found positive predictive values of 63-90\% and negative predictive values of 66-89\% (16-18). Table 2 shows the different testing characteristics of various testing modalities used in the CKD and dialysis population.

A meta-analysis assessed the prognostic utility of myocardial perfusion studies (which included both stress echo and thallium scintigraphy). For diabetic and non-diabetic ESRD and CKD patients evaluated for transplant, the presence of reversible defects was associated with a 6-fold increased risk for MI and a 4 fold increased risk for cardiac death. Of note, diabetic patients with a negative study still had a $2.4 \%$ annual incidence of MI and $0.9 \%$ incidence of cardiac death. The authors concluded that these are good tests that predict both angiographically significant coronary artery disease, future MI and cardiac death (19). Overall, dobutamine stress echocardiography and dipyridamole thallium myocardial scintigraphy appear to be good initial tests in a pre-operative evaluation.

Some would advocate that coronary angiography is a better predictor of later cardiac events. One study compared non-invasive testing, risk stratification and coronary angiography in kidney transplant candidates over 2 years. The researchers found that coronary angiography was the best predictor of survival. Those who had $<70 \%$ coronary artery stenosis on angiography had a 48 month cardiac event free survival of $94 \%$ while those with $>70 \%$ stenosis had a 48 month cardiac event free survival of $54 \%$. They concluded that coronary angiography may be better for high risk renal transplant candidates (20). A study comparing medical management to coronary revascularization in asymptomatic diabetic patients concluded that coronary revascularization prior to transplantation potentially decreases the risk of myocardial infarction post-transplant in insulin dependent diabetics (mostly type I) with chronic kidney disease (21). 
Significant concern exists for contrast nephropathy after coronary angiography in stage 4 and 5 CKD patients .. A recent retrospective study evaluated the risk of chronic kidney disease progression after coronary angiography (22). In this study the patients received prophylactic measures including small volumes of contrast, $\mathrm{N}$-acetylcysteine, intravenous fluids and avoidance of high risk medications prior to the procedure (22). Although this study was retrospective with concern for confounding, the authors found that the exposure to contrast in this population was tolerated relatively well, with $89 \%$ of patients remaining dialysis free at six months post coronary angiography (22). However, with the increased risk of morbidity and mortality with revascularization procedures (both heart catheterizations and coronary bypass surgery), as well as postponement of the kidney transplant surgery, one must weigh the risks and benefits of performing these more invasive procedures as part of a pre-transplant work-up. This approach increases the need to accurately and reliably identify high risk patients using a risk score who would likely benefit the most from cardiac revascularization and closer follow-up for modifiable coronary artery disease risk factors.

Given the above pros and cons of pre-kidney transplant testing, we describe a possible algorithm based on synthesis of available literature for the pre-transplant cardiac evaluation in Figure 1. This combines both a risk score and cardiac evaluation schema, which could also help maximize cost efficiency in pre-transplant evaluations by avoiding the need for testing in very low cardiac risk patients. The risk score would designate patients into a low, intermediate, and high risk category. Low risk patients would not require pre-transplant cardiac testing, high risk patients would directly undergo heart catheterization, while the intermediate group would undergo non-invasive stress echo or nuclear stress. If the noninvasive study is positive, the patient undergoes catheterization; if negative the patient is listed for kidney transplantation. Patients who undergo catheterization get revascularization if indicated prior to listing. When applied to our patients: if patient 1 had a high risk score, he would have had a cardiac catheterization with intervention prior to surgery; patient 2 may have avoided an invasive cardiac catheterization with a low risk score and could have had continued aggressive risk modification prior to and after surgery. In low risk CKD patients, aggressive risk modification is preferable to invasive procedures requiring exposure to iodinated contrast and the risk for loss of residual renal function. Future prospective investigations are still necessary, with the aim of randomizing patients to different cardiac evaluation algorithms pre-transplant and follow them for cardiac events.

The utility of non-kidney transplant specific risk scores in identifying which patients are at highest risk of a cardiovascular event prior to and after transplantation have been evaluated using previously defined methods. In one study, pre-transplant cardiac testing was not done in $44 \%$ of their study population that they considered to be low risk (23). Given that this was a retrospective study, however, the specific criteria used to classify each patient as high or low risk was not necessarily uniform. Others have used the well-established Framingham risk score to determine its ability to predict cardiac events post-kidney transplant. These studies found that the Framingham score tended to underestimate the risk of cardiac events post-kidney transplant, specifically for the diabetic transplant patient population $(24,25)$. These studies are consistent with the finding that already existing risk scores do not adequately predict cardiac events post-transplantation, and encourages further investigations aimed at creating a risk score that would better predict cardiac events in kidney transplant patients.

A risk score has been developed specifically for overall survival in potential kidney transplant recipients (26). In this study, angina was the only cardiac variable included but had one of the highest hazard ratios (26). Investigators from an ongoing cohort study in post-transplant patients from the placebo arm of the ALERT trial are attempting to create a prediction model for major adverse cardiac events (27). Another group is currently 
evaluating the efficacy and costs of screening patients undergoing living donor transplants who are asymptomatic, though high risk for cardiac events, using standard evaluation and screening tests or according to ACC guidelines for non-cardiac surgery (28).

\section{Conclusions}

The goal of a cardiac prediction score in the pre-kidney transplant population is the accurate and reliable prediction of peri-operative and post-operative cardiovascular morbidity and mortality. Such a score could guide the use of cardiac screening and interventions prior to surgery with the aim of minimizing insults to newly transplanted kidneys, avoiding potentially harmful tests and procedures, and maximizing the patient's allograft, and overall, survival. A scoring system of this type would have benefited both of our patients, as one suffered a cardiac event within two weeks of transplant that resulted in a coronary artery bypass graft surgery, and the other underwent an invasive procedure which was potentially avoidable were she considered a low risk candidate. Future prospective investigations, preferably randomized control trials, are needed to look at the different pre-transplant algorithms, such as the ASN/AST and ACC/AHA guidelines, as they apply to our patients.

\section{The Training Program in Nephrology at Oregon Health and Science University School of Medicine}

Postdoctoral training in nephrology at the Oregon Health and Science University is a major focus of the Division of Nephrology and Hypertension. The program is currently directed by Suzanne Watnick, M.D. and consists of two pathways. The Clinical Pathway is a 2-year program, designed for fellows with an interest in a clinical practice career. For this pathway, approximately 15-18 months are clinical training, and 6-9 months are dedicated to research and scholarship. The Research Pathway is designed for fellows with an interest in an academic career, with development of skills in education and research (either basic science or clinical). This is a 3-year program, with approximately 12-13 clinical months and the remainder dedicated to research and scholarship.

The clinical experience occurs primarily at the University Hospital and the Portland VA Medical Center. Experiences are provided in acute and chronic hemodialysis, peritoneal dialysis, renal transplantation, renal biopsy, and continuous renal replacement therapy, as well as in the interpretation of renal pathology specimens and radiographs. The development of diagnostic skills in renal disease, hypertension, and acid-base, fluid and electrolyte disorders is of primary importance in the training program. The fellow will receive extensive experience in patient evaluation, diagnosis, and treatment design.

In addition to inpatient responsibilities, fellows participate in the hypertension, transplantation and nephrology outpatient clinics of the division, and the outpatient chronic hemodialysis and peritoneal dialysis programs. Fellows are supervised by a faculty member on each rotation, and are responsible for the supervision of house officers and medical students.

Research training is the focus of the second and third years and is designed to develop the ability to become an independent investigator. With direction from the Division Head (David H. Ellison, M.D.), Fellowship Director (Suzanne Watnick, M.D.), and Director of Fellowship Research (David Cohen, M.D.), the fellow will select an area of research interest and a faculty preceptor for accomplishing his or her research objectives. There is opportunity to participate on our T-32 Training grant. During research months, fellows will have limited clinical responsibility, usually consisting of a weekly outpatient clinic and/or continuity dialysis patients. The remainder of the fellow's time is focused on 
research projects, and continued participation in the educational conferences of the division.

The Division of Nephrology and Hypertension consists of 17 full- and part-time faculty. Basic research interests include mechanisms and regulation of epithelial transport, glomerular hemodynamic factors in disease progression; diabetic nephropathy; the role of various vasoactive hormones in modulation renal function; fetal origins of hypertension; and biochemical and molecular mechanisms of signaling pathways and gene transcription. Clinical research interests include the study of biomarkers to identify toxic injury to the kidneys, inherited disorders of the kidney, outcomes after renal transplantation, and quality of life issues with respect to end-stage renal disease.

Further information regarding the nephrology fellowship training program at OHSU can be obtained on our website: www.ohsu.edu/som-nephrology.

\section{Acknowledgments}

This work was supported by NIH/NIDDK grant T32 DK067864

\section{References}

1. Wolfe RA, Ashby VB, Milford EL, Ojo AO, Ettenger RE, Agodoa LY, Held PJ, Port FK. Comparison of mortality in all patients on dialysis, patients on dialysis awaiting transplantation, and recipients of a first cadaveric transplant. N Engl J Med. 1999; 341:1725-1730. [PubMed: 10580071]

2. Scientific registry of transplant recipients. 20092009.

3. U.S. renal data system. USRDS 2006 annual data report: Atlas of end-stage Renal disease in the united states, national institutes of Health, national institute of diabetes and digestive and kidney diseases. Bethesda, MD: 2009. 2008

4. Kasiske BL, Cangro CB, Hariharan S, Hricik DE, Kerman RH, Roth D, Rush DN, Vazquez MA, Weir MR. American Society of T. The evaluation of renal transplantation candidates: Clinical practice guidelines. Am J Transplant. 2001; 1:3-95. [PubMed: 12108435]

5. Fletcher GF, Balady GJ, Amsterdam EA, Chaitman B, Eckel R, Fleg J, Froelicher VF, Leon AS, Pina IL, Rodney R, Simons-Morton DA, Williams MA, Bazzarre T. Exercise standards for testing and training: A statement for healthcare professionals from the american heart association. Circulation. 2001; 104:1694-1740. [PubMed: 11581152]

6. Fleisher LA, Beckman JA, Brown KA, Calkins H, Chaikof E, Fleischmann KE, Freeman WK, Froehlich JB, Kasper EK, Kersten JR, Riegel B, Robb JF, Smith SC Jr, Jacobs AK, Adams CD, Anderson JL, Antman EM, Buller CE, Creager MA, Ettinger SM, Faxon DP, Fuster V, Halperin JL, Hiratzka LF, Hunt SA, Lytle BW, Md RN, Ornato JP, Page RL, Riegel B, Tarkington LG, Yancy CW. ACC/AHA TASK FORCE MEMBERS. ACC/AHA 2007 guidelines on perioperative cardiovascular evaluation and care for noncardiac surgery: Executive summary: A report of the american college of Cardiology/American heart association task force on practice guidelines (writing committee to revise the 2002 guidelines on perioperative cardiovascular evaluation for noncardiac surgery): Developed in collaboration with the american society of echocardiography, american society of nuclear cardiology, heart rhythm society, society of cardiovascular anesthesiologists, society for cardiovascular angiography and interventions, society for vascular medicine and biology, and society for vascular surgery. Circulation. 2007; 116:1971-1996. [PubMed: 17901356]

7. Ojo AO, Hanson JA, Wolfe RA, Leichtman AB, Agodoa LY, Port FK. Long-term survival in renal transplant recipients with graft function. Kidney Int. 2000; 57:307-313. [PubMed: 10620213]

8. Foley RN, Parfrey PS, Sarnak MJ. Epidemiology of cardiovascular disease in chronic renal disease. J Am Soc Nephrol. 1998; 9:S16-S23. [PubMed: 11443763]

9. Parfrey PS, Foley RN. The clinical epidemiology of cardiac disease in chronic renal failure. J Am Soc Nephrol. 1999; 10:1606-1615. [PubMed: 10405218] 
10. Parfrey PS. Cardiac disease in dialysis patients: Diagnosis, burden of disease, prognosis, risk factors and management. Nephrol Dial Transplant. 2000; 15:58-68. [PubMed: 11073277]

11. Ohtake T, Kobayashi S, Moriya H, Negishi K, Okamoto K, Maesato K, Saito S. High prevalence of occult coronary artery stenosis in patients with chronic kidney disease at the initiation of renal replacement therapy: An angiographic examination. J Am Soc Nephrol. 2005; 16:1141-1148. [PubMed: 15743997]

12. Gradaus F, Ivens K, Peters AJ, Heering P, Schoebel FC, Grabensee B, Strauer BE. Angiographic progression of coronary artery disease in patients with end-stage renal disease. Nephrol Dial Transplant. 2001; 16:1198-1202. [PubMed: 11390720]

13. Lewis MS, Wilson RA, Walker KW, Wilson DJ, Norman DJ, Barry JM, Bennett WM. Validation of an algorithm for predicting cardiac events in renal transplant candidates. Am J Cardiol. 2002; 89:847-850. [PubMed: 11909572]

14. Marwick TH, Steinmuller DR, Underwood DA, Hobbs RE, Go RT, Swift C, Braun WE. Ineffectiveness of dipyridamole spect thallium imaging as a screening technique for coronary artery disease in patients with end-stage renal failure. Transplantation. 1990; 49:100-102. [PubMed: 2300998]

15. Schmidt A, Stefenelli T, Schuster E, Mayer G. Informational contribution of noninvasive screening tests for coronary artery disease in patients on chronic renal replacement therapy. Am J Kidney Dis. 2001; 37:56-63. [PubMed: 11136168]

16. Gang S, Dabhi M, Rajapurkar MM. Ischaemia imaging in type 2 diabetic kidney transplant candidates--is coronary angiography essential? Nephrol Dial Transplant. 2007; 22:2334-2338. [PubMed: 17550928]

17. Sharma R, Pellerin D, Gaze DC, Gregson H, Streather CP, Collinson PO, Brecker SJ. Dobutamine stress echocardiography and the resting but not exercise electrocardiograph predict severe coronary artery disease in renal transplant candidates. Nephrol Dial Transplant. 2005; 20:22072214. [PubMed: 16030034]

18. Ferreira PA, de Lima VC, Campos Filho O, Gil MA, Cordovil A, Machado CV, Pestana JO, Carvalho AC. Feasibility, safety and accuracy of dobutamine/atropine stress echocardiography for the detection of coronary artery disease in renal transplant candidates. Arq Bras Cardiol. 2007; 88:45-51. [PubMed: 17364118]

19. Rabbat CG, Treleaven DJ, Russell JD, Ludwin D, Cook DJ. Prognostic value of myocardial perfusion studies in patients with end-stage renal disease assessed for kidney or kidney-pancreas transplantation: A meta-analysis. J Am Soc Nephrol. 2003; 14:431-439. [PubMed: 12538744]

20. De Lima JJ, Sabbaga E, Vieira ML, de Paula FJ, Ianhez LE, Krieger EM, Ramires JA. Coronary angiography is the best predictor of events in renal transplant candidates compared with noninvasive testing. Hypertension. 2003; 42:263-268. [PubMed: 12913060]

21. Manske CL, Wang Y, Rector T, Wilson RF, White CW. Coronary revascularisation in insulindependent diabetic patients with chronic renal failure. Lancet. 1992; 340:998-1002. [PubMed: 1357450]

22. Kumar N, Dahri L, Brown W, Duncan N, Singh S, Baker C, Malik I, Palmer A, Griffith M, Cairns T, Taube D. Effect of elective coronary angiography on glomerular filtration rate in patients with advanced chronic kidney disease. Clin J Am Soc Nephrol. 2009; 4:1907-1913. [PubMed: 19833903]

23. Kasiske BL, Malik MA, Herzog CA. Risk-stratified screening for ischemic heart disease in kidney transplant candidates. Transplantation. 2005; 80:815-820. [PubMed: 16210970]

24. Kasiske BL, Chakkera HA, Roel J. Explained and unexplained ischemic heart disease risk after renal transplantation. J Am Soc Nephrol. 2000; 11:1735-1743. [PubMed: 10966499]

25. Kiberd B, Panek R. Cardiovascular outcomes in the outpatient kidney transplant clinic: The framingham risk score revisited. Clin J Am Soc Nephrol. 2008; 3:822-828. [PubMed: 18322053]

26. Baskin-Bey ES, Kremers W, Nyberg SL. A recipient risk score for deceased donor renal allocation. Am J Kidney Dis. 2007; 49:284-293. [PubMed: 17261431]

27. Fellstrom BC, Holdaas H, Jardine A. Cardiovascular risk calculator in renal transplantation. ASN. 2007:SA-PO425. (Abstract). 
28. Kasiske, BL. The coronary screening for kidney transplantation (COST) study. Organization: University of Minnesota Twin Cities; 2008. National Institute of Diabetes and Digestive and Kidney Diseases. (Electronic version) Project number: 5R21DK080315-02 


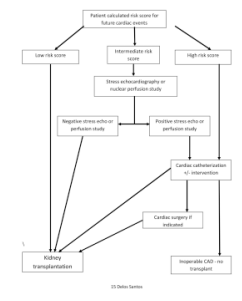

Figure 1.

Proposed pre-transplant cardiac evaluation algorithm. A calculated risk score designed and validated in the kidney transplant population can be integrated into a cardiac evaluation schema. The risk score would designate patients into a low, intermediate, and high risk category. Following the schema low risk patients can be listed without additional testing. High risk patients undergo cardiac catheterization. Intermediate risk patients undergo noninvasive testing and have cardiac catheterization if the non-invasive testing is positive for ischemia. 
Table 1

Patient 1 and 2 pre-transplant characteristics compared

\begin{tabular}{|c|c|c|}
\hline & Patient 1 & Patient 2 \\
\hline \multicolumn{3}{|l|}{ Past medical history of: } \\
\hline Hypertension & yes & yes \\
\hline Hyperlipidemia & yes & yes \\
\hline Coronary artery disease & yes (with stents) & no \\
\hline Smoking history & yes & no \\
\hline \multicolumn{3}{|l|}{ Family history of: } \\
\hline Diabetes & no & yes \\
\hline Cardiovascular disease & yes & yes \\
\hline \multicolumn{3}{|l|}{ Medications: } \\
\hline Beta blocker & yes & yes \\
\hline Calcium channel blocker & yes & yes \\
\hline Angiotensin converting enzyme inhibitor & yes & no \\
\hline HMG-CoA reductase inhibitor (statin) & yes & yes \\
\hline Aspirin & yes & no \\
\hline Insulin & no & yes \\
\hline
\end{tabular}


Table 2

Cardiac testing modalities for the presence of coronary artery disease: sensitivities, specificities, positive predictive value and negative predictive value

\begin{tabular}{|lcccc|}
\hline Cardiac test & Sens (\%) & Spec (\%) & PPV (\%) & NPV (\%) \\
\hline Resting ECG & $67-77$ & $52-58$ & 43 & 47 \\
\hline Exercise stress & 35 & 64 & $63^{*}$ & $36 *$ \\
\hline Stress echocardiography & $47-88$ & $85-95$ & $63-90$ & $66-89$ \\
\hline Nuclear dipyridamole & $37-80$ & $37-73$ & $53-58$ & $60-67$ \\
\hline
\end{tabular}

Various ranges for the sensitivities, specificities, positive predictive values and negative predictive values are listed above for the different stress testing modalities in the chronic kidney disease or dialysis population. Sens $=$ sensitivity, Spec $=$ specificity, PPV $=$ positive predictive value, NPV $=$ negative predictive value.

n.b. - PPV and NPV were calculated by the authors using a $64 \%$ prevalence of cardiac disease 